\title{
HERA PDFs
}

\section{Ringailè Plačakyté}

on behalf of the HI and ZEUS Collaborations

Deutsches Elektronen-Synchrotron DESY,

Notkestr. 85, 22607 Hamburg, Germany.

E-mail: ringaileemail.desy.de

The parton distribution functions (PDFs) of the proton are necessary non-perturbative inputs for calculating almost all scattering processes at hadron colliders. An overview of the HERA PDF sets determined by the H1 and ZEUS collaborations and an open source project for PDF determination, HERAFitter, are presented in this report.

36th International Conference on High Energy Physics

4-11 July 2012

Melbourne, Australia

${ }^{*}$ Speaker. 


\section{Introduction}

The cross sections of various scattering processes at hadron colliders are calculated by convoluting the parton level cross sections with the parton distribution functions (PDFs). Generally, the parton distribution function $f_{i}\left(x, Q^{2}\right)$ gives the probability of finding a parton (quark or gluon) of flavour $i$ in the proton carrying a fraction $x$ of the proton momentum with $Q$ being the energy scale of the hard interaction. PDFs cannot be predicted by perturbative QCD. They are determined through a fit procedure of experimental data to the theoretical models using the DGLAP evolution equation [四]. The knowledge of proton PDFs mainly comes from Deep Inelastic Scattering (DIS) at HERA and at fixed target experiments and from TEVATRON data. The LHC data has the potential to improve the understanding of PDFs further.

Presently, the determination of PDFs is mainly carried out by several groups, namely MSTW [2],

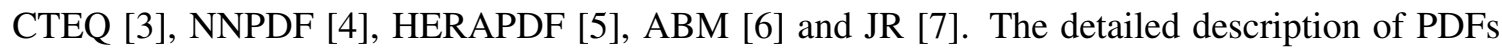
from the HERAPDF group is provided in this write-up.

\section{HERAPDF sets}

The HERAPDF sets are extracted primarily from the inclusive neutral current (NC) and charged current (CC) deep inelastic scattering data measured at the HERA collider. At HERA electrons (or positrons) were collided with protons at centre-of-mass energies $\sqrt{s}=225-318 \mathrm{GeV}$. In neutral current reactions, $e p \rightarrow e X$, the interaction proceeds via the exchange of a photon or $\mathrm{Z}$ boson while in charged current scattering, $e p \rightarrow v X$, a $W^{ \pm}$boson is exchanged. The NC (and similarly $\mathrm{CC})$ cross section can be expressed in terms of structure functions:

$$
\frac{d^{2} \sigma_{N C}^{e^{ \pm} p}}{d x d Q^{2}}=\frac{2 \pi \alpha^{2}}{x Q^{4}}\left[Y_{+} \tilde{F}_{2}^{ \pm} \mp Y_{-} x \tilde{F}_{3}^{ \pm}-y^{2} \tilde{F}_{L}^{ \pm}\right]
$$

where $Y_{ \pm}=1 \pm(1-y)^{2}$, with $y$ characterising the inelasticity of the interaction. The structure function $\tilde{F}_{2}$ is the dominant contribution to the cross section, $x \tilde{F}_{3}$ is important at high $Q^{2}$ and $\tilde{F}_{L}$ is sizable only at high $y$. In the framework of perturbative QCD the structure functions are directly related to the parton distribution functions, i.e. in leading order (LO) $\tilde{F}_{2}$ is determined by the sum of quark and anti-quark momentum distributions while the structure function $x \tilde{F}_{3}$ is related to their difference. At higher orders, terms related to the gluon distribution function $\left(\alpha_{s} g\right)$ appear.

The inclusive CC ep cross section can also be expressed in terms of parton distributions. In the LO the $e^{+} p$ and $e^{-} p$ cross sections are sensitive to different quark densities:

$$
\begin{array}{ll}
e^{+}: & \tilde{\sigma}_{C C}^{e^{+} p}=x[\bar{u}+\bar{c}]+(1-y)^{2} x[d+s] \\
e^{-}: & \tilde{\sigma}_{C C}^{e^{-} p}=x[u+c]+(1-y)^{2} x[\bar{d}+\bar{s}] .
\end{array}
$$

In addition to NC and CC scattering measured at HERA, some variants of HERAPDF sets also use the jet and charm measurements at HERA. The existing HERAPDF sets are summarised in table $\square$ and described in details below.

In the HERAPDF1.0 fit [ [ $]$, the HERA combined measurement of the inclusive NC and CC ep cross sections from the first running period of HERA (HERA-I) is used. The method used in the data combination takes into account the correlations of systematic uncertainties resulting in an 


\begin{tabular}{|l|l|l|}
\hline \hline PDF set name & DATA & Order \\
\hline \hline HERAPDF1.0 & H1-ZEUS CC,NC HERA-I & NLO, NNLO \\
\hline HERAPDF1.5 & H1-ZEUS CC,NC HERA-I and HERA-II(prel.) & NLO, NNLO \\
\hline HERAPDF1.6 & H1-ZEUS CC,NC HERA-I and HERA-II(prel.) and jets & NLO \\
\hline HERAPDF1.0+charm & H1-ZEUS CC,NC HERA-I and charm & NLO \\
\hline HERAPDF1.7 & All data above & NLO \\
\hline \hline
\end{tabular}

Table 1: List of HERAPDF parton distribution function sets. HERAPDF1.0 and HERAPDF1.5 sets with the full uncertainty estimation are available through the Les Houches Accord PDF Interface (LHAPDF).

improved accuracy.

The QCD predictions for the structure functions are obtained by solving the DGLAP evolution equations at NLO (or NNLO) in the $\overline{\mathrm{MS}}$ scheme with the renormalisation and factorisation scales chosen to be $Q^{2}$. The DGLAP equations yield the PDFs at all values of $Q^{2}$ above the input scale $Q_{0}^{2}$ at which they are parametrised as a function of $x$. The starting scale $Q_{0}^{2}$ is chosen to be $1.9 \mathrm{GeV}^{2}$ such that it is below the charm mass threshold. The heavy quark coefficient functions are calculated in the RT general mass variable favour number scheme [ [ $]$ ].

The generic form of the parametrisation is used at the input scale. The parametrised PDFs are the gluon distribution $x g$, the valence quark distributions $x u_{v}, x d_{v}$, and the $u$-type and $d$-type anti-quark distributions $x \bar{U}, x \bar{D}$. At the starting scale $Q_{0}^{2}=1.9 \mathrm{GeV}^{2} x \bar{U}=x \bar{u}$ and $x \bar{D}=x \bar{d}+x \bar{s}$. The central fit parametrisation is:

$$
\begin{aligned}
x g(x) & =A_{g} x^{B_{g}}(1-x)^{C_{g}}, \\
x u_{v}(x) & =A_{u_{v}} x^{B_{u_{v}}}(1-x)^{C_{u_{v}}}\left(1+E_{u_{v}} x^{2}\right), \\
x d_{v}(x) & =A_{d_{v}} x^{B_{d_{v}}}(1-x)^{C_{d_{v}}}, \\
x \bar{U}(x) & =A_{\bar{U}} x^{B_{\bar{U}}}\left(1-x C_{\bar{U}}\right), \\
x \bar{D}(x) & =A_{\bar{D}} x^{B_{\bar{D}}}\left(1-x^{C_{\bar{D}}}\right) .
\end{aligned}
$$

The normalisation parameters $A$ are constrained by the quark number sum-rules and momentum sum-rule, extra constrains are applied for the small- $x$ behaviour of $d$ - and $u$-type quarks $B_{u_{v}}=B_{d_{v}}$, $B_{\bar{U}}=B_{\bar{D}}$ and $A_{\bar{U}}=A_{\bar{D}}\left(1-f_{s}\right)\left(f_{s}\right.$ is the strange quark fraction) which ensures that $x \bar{u} \rightarrow x \bar{d}$ as $x \rightarrow 0$.

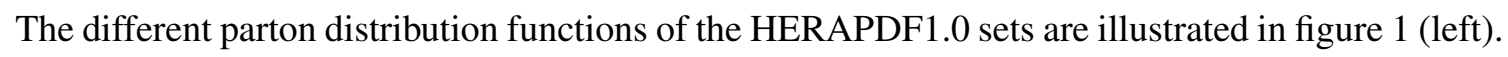
Model uncertainties (shown as yellow bands in the figure) of the central fit solution are evaluated by varying the input assumptions: $Q_{\min }^{2}, f_{s}$, masses of the heavy quarks $m_{C}$ and $m_{B}$. The parametrisation uncertainty (green band) is formed by an envelope of the maximal deviation from the central fit, varying parametrisation assumptions in the fit (by adding additional parameters to (ㄹ.3]) ) and therefore has an asymmetric shape. The determination of parameterisation uncertainties is unique to the HERAPDF sets.

The full statistics HERA (HERA-I and HERA-II) inclusive CC and NC data are used for NLO and NNLO QCD fits resulting in HERAPDF1.5 set [Q]. The same formalism, model and paramatrisation assumptions as for the HERAPDF1.0 are used in the HERAPDF1.5(NLO) fit. As can be seen in figure $\mathbb{M}$, the improved statistics of the inclusive HERA data results in reduced experimental and model uncertainties of the PDFs, especially for valence quark distributions, driven by the more 

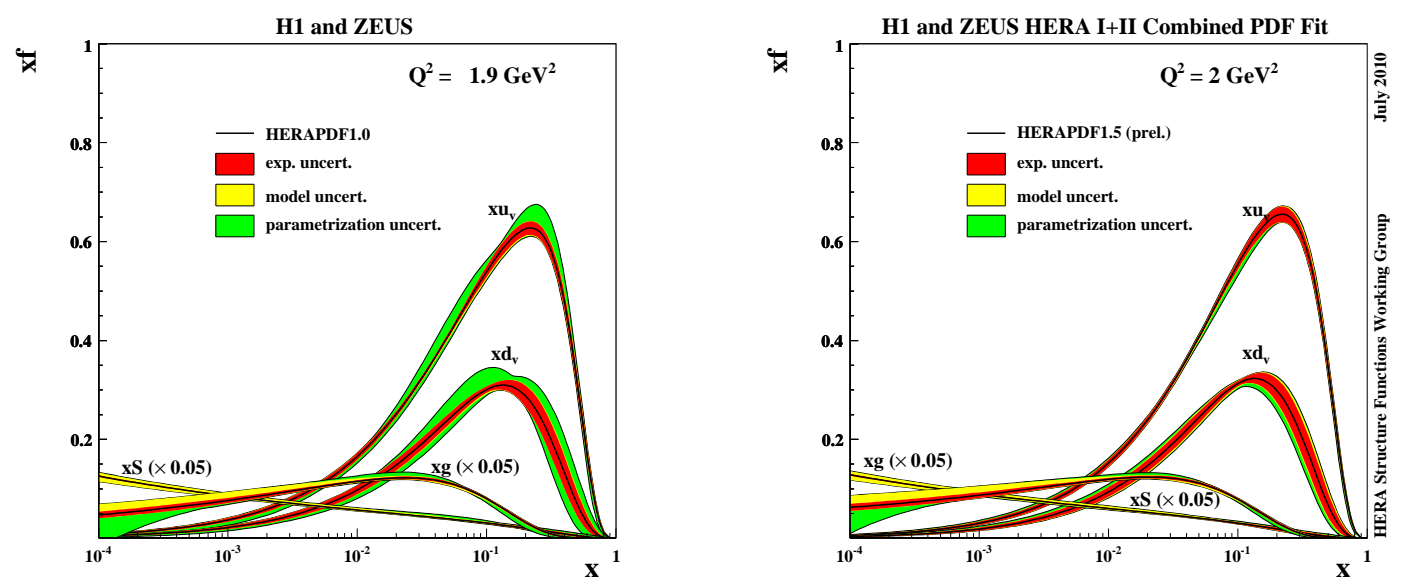

Figure 1: The parton distribution functions from HERAPDF1.0 (left) and HERAPDF1.5 (right) shown for $x u_{v}, x d_{v}, x S=2 x(\bar{U}+\bar{D}), x_{g}$ at $Q^{2}=1.9 \mathrm{GeV}^{2}$. The gluon and sea distributions are scaled down by a factor 20. The different color bands represent experimental, model and parametrisation uncertainties, respectively.

precise CC data.

The HERAPDF1.5 analysis has been extended to include HERA inclusive jet data resulting to the HERAPDF1.6 [ए]] set. The jet data are sensitive to gluon and quark distributions in the PDFs and to the strong coupling constant $\alpha_{s}$. The impact of the jet data can be seen if $\alpha_{s}\left(M_{Z}\right)$ is treated as a free parameter in the fit (figure $\square$ ). In case of fitting simultaneously PDFs and $\alpha_{s}$ to only the DIS data the uncertainties on the gluon PDF becomes large at low $x$ (figure $\square$ left). By including the jet data the correlation between the gluon PDF and $\alpha_{s}\left(M_{Z}\right)$ is reduced, resulting in significantly smaller uncertainties on the gluon PDF (figure $\square$ right) which are similar in size to those obtained by using inclusive DIS data only and fixing $\alpha_{s}\left(M_{Z}\right)$. In the fit with jet data the value of extracted $\alpha_{s}\left(M_{Z}\right)=0.1202 \pm 0.0013(\exp ) \pm 0.0007(\mathrm{mod} /$ param $) \pm 0.0012(\mathrm{had}){ }_{-0.0036}^{+0.0045}(\mathrm{th})$ [ய0] .
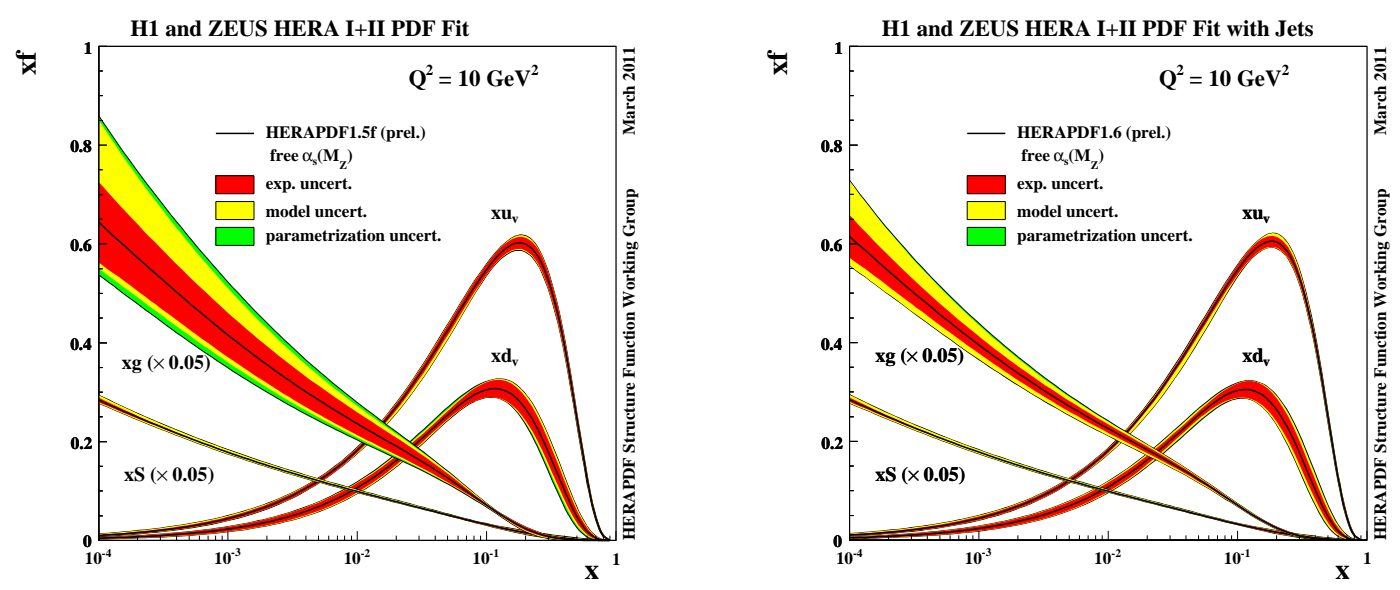

Figure 2: Parton distribution functions for HERAPDF1.5 without (left) and with jet data (right) treating $\alpha_{s}$ as a free parameter in the fit as a function of $x$ for $Q^{2}=10 \mathrm{GeV}^{2}$. The central value (solid line) is shown together with experimental, model and parametrisation uncertainties represented by colored bands. 
The effect of including the HERA charm production data in the PDF fits is also studied [U]]. In this study, several implementations of the variable flavour number schemes (VFNS), which have different treatment of heavy quark mass terms in $\mathrm{pQCD}$, are investigated. The different VFN schemes can have different impacts on the charm contribution to the sea quark and thus affect the composition of $x \bar{U}(x)$ from the $x \bar{u}(x)$ and the $x \bar{c}(x)$ contributions. In the study, a combined NLO QCD analysis is performed using the charm production and the inclusive DIS data repeatedly for each VFN scheme with different values of the charm quark mass parameter, $m_{c}^{\text {model }}$. Figure B (left) shows the results of the $m_{c}^{\text {model }}$ scan for all schemes considered. It is interesting to observe that first, different schemes have different optimal charm quark mass parameter $m_{c}^{\text {model }}$, and second, the $\chi^{2}$ minimum values are comparable for all schemes despite of different optimal values of $m_{c}^{\text {model }}$. The implications of this result on the NLO predictions for $W^{+}$and $Z$ production cross sections at the LHC are investigated by calculating the boson cross sections with PDF sets produced for each scheme at its optimal $m_{c}^{\text {model }}$. As an example, the $W^{+}$cross section as a function of $m_{c}^{\text {model }}$ for the different schemes is shown in figure B (right). Good agreement between these predictions is observed at optimal $m_{c}^{\text {model }}$ which results in a reduction of the uncertainties due to the heavy flavour treatment to below $1.0 \%$.
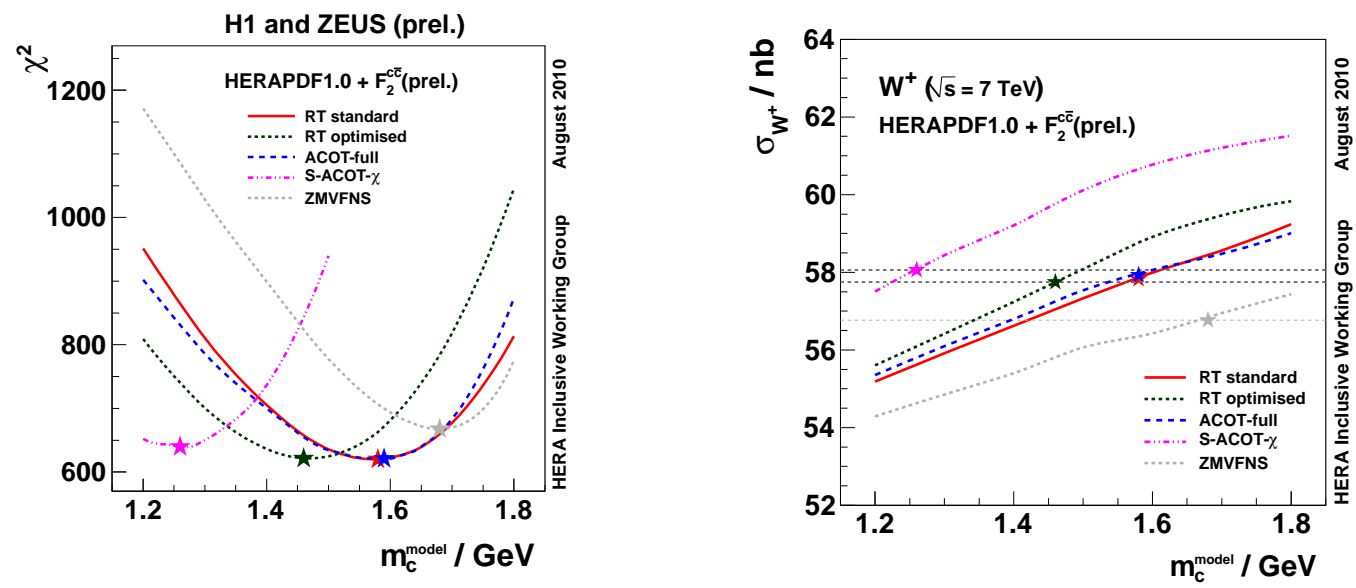

Figure 3: Left: Comparison of the $\chi^{2}$ obtained by NLO fits to the inclusive HERA-I and the preliminary combined charm data in different VFN schemes represented as lines of different styles. Right: $W^{+}$production cross section $\sigma_{W^{+}}$at the $\mathrm{LHC}$ for $\sqrt{s}=7 \mathrm{TeV}$ as a function of $m_{c}^{\text {model }}$. Lines represent different schemes and the stars show the positions of the corresponding optimal $m_{c}^{\text {model }}$ values obtained for each scheme.

Finally, a QCD analysis is performed which includes all currently available HERA NC and CC inclusive, charm, low energy and jet data (HERAPDF1.7). This fit prefers the same $\alpha_{s}$ value as obtained with a fit using only inclusive and jet data. Similarly, the same optimal $m_{c}^{\text {model }}$ value was found in the HERAPDF1.7 fit as that obtained in a fit when only inclusive and charm data are fitted.

\section{HERAFitter project}

The HERAFitter project is a common initiative of the HERA experiments joined by the LHC and theory groups to provide an open source framework containing PDF fitting tools [ए2]. Although the fitting framework was initially used for the HERAPDF fitts, it has since evolved and 
incorporates different type of data and is actively used by the LHC community. These include inclusive cross sections from HERA DIS and fixed target experiments, Drell-Yan, jet production data $(e p, p p$ and $p \bar{p}$ ) and heavy quark structure functions. Experimental systematic uncertainties can be treated as correlated or uncorrelated and there are several methods provided how to calculate them (Hessian covariance matrix, Monte Carlo replicas). The structure functions may be computed in a variety of heavy quark schemes as well as the fixed flavour scheme. The package is interfaced to different tools like FastNLO and APPLGRID for the fast input of NLO jet and Drell-Yan cross sections and HATHOR for the calculation of the top cross sections. HERAFitter is under continues development and currently includes many useful additions. Basic plots are provided together with the fit result and the resulting PDFs are supported by the LHAPDF standards.

In summary, HERAFitter contains all necessary ingredients to study the proton PDFs, it incorporates variety of different data processes and theory calculations, contains many useful tools and is an optimal platform for various benchmarking studies.

\section{References}

[1] V.N. Gribov, L.N. Lipatov, Sov. J. Nucl. Phys. 15, 438, 675 (1972), L.N. Lipatov, Sov. J. Nucl. Phys. 20, 94 (1975), G. Altarelli, G. Parisi, Nucl. Phys. B126, 298 (1977), Yu. L. Dokshitzer, Sov. Phys. JETP 46, 641 (1977), G. Curci, W. Furmanski, and R. Petronzio, Nucl.Phys. B175, 27 (1980), S. Moch, J. Vermaseren, and A. Vogt, Nucl.Phys. B688, 101 (2004) [arXiv:hep-ph/0403192], A. Vogt, S. Moch, and J. Vermaseren, Nucl.Phys. B691, 129 (2004) [arXiv:hep-ph/0404111].

[2] A.D. Martin, W.J. Stirling, R.S. Thorne, G. Watt, Eur. Phys. J. C63, 189 (2009) [arXiv:0901.0002].

[3] Hung-Liang Lai et al., Phys. Rev. D82, 074024 (2010), [arXiv:1007.2241], P. M. Nadolsky et al, PoS DIS2012 (2012) [arXiv:1206.3321].

[4] Richard D. Ball et al., Nucl. Phys. B849 296 (2011), [arXiv:1101.1300], Richard D. Ball et al., Nucl. Phys. B855 153 (2012), [arXiv:1107.2652],

[5] F. Aaron at al. [H1 and ZEUS Collaborations], JHEP B1001, 109 (2010) [0911.0884].

[6] S. Alekhin, J. Blümlein, S. Klein, S. Moch, Phys. Rev. D81, 014032 (2010) [arXiv:0908.2766], S. Alekhin, J. Blümlein, S. Moch, PoS DIS2010 (2010) [arXiv:1007.3657], S. Alekhin, J. Blümlein, S. Moch, Phys.Rev. D86 (2012) [arXiv:1202.2281].

[7] M. Glück, P. Jimenez-Delgado, E. Reya, Eur. Phys. J. C53, 355 (2008) [arXiv:0709.0614], M. Glück, P. Jimenez-Delgado, E. Reya, C. Schuck, Phys. Lett. B664, 133 (2008) [arXiv:0801.3618].

[8] R. S. Thorne and R. G. Roberts, Phys. Rev. D57, 6871 (1998) [hep-ph/9709442].

[9] [H1 and ZEUS Collaborations], H1prelim-10-142, ZEUS-prel-10-018, [H1 and ZEUS Collaborations], H1prelim-11-042, ZEUS-prel-11-002.

[10] [H1 and ZEUS Collaborations], H1prelim-11-034, ZEUS-prel-11-001.

[11] [H1 and ZEUS Collaborations], H1prelim-10-143, ZEUS-prel-10-019.

[12] HERAFitter, http://projects.hepforge.org/heraftter,

F. D. Aaron et al. (H1 and ZEUS Collaborations), JHEP 01, 109 (2010), F. Aaron et al. (H1 Collaboration), Eur. Phys. J. C64, 561 (2009), M. Botje, Comput. Phys. Commun. 182, 490 (2011). 